\title{
Accessing Cause-Result Relation and Diplomatic Information in Ancient "Journalistic" Texts with Universal Words
}

\author{
Christina Alexandris \\ National University of Athens, Greece \\ calexandris@gs.uoa.gr
}

\begin{abstract}
For the International Public, ancient historical and "journalistic" texts, such the "Peloponnesian War" of the Ancient Greek historian Thucydides, may allow an insight for the understanding of current international political and economic relations. The present approach targets to facilitate the accessibility of such texts for non-experts in the International Public, with no knowledge of the ancient language concerned, especially journalists, translators and students. The possibility of directly accessing text content and viewing features, as close as possible to the original text is attempted to be achieved here, using predefined sublanguage-specific keywords and Universal Words.
\end{abstract}

Keywords: Ancient Greek, keyword ontology, Universal Words, International Public, online Machine Translation.

\section{Introduction and User Requirements}

For the International Public, ancient historical and "journalistic" texts, such the "Peloponnesian War" of the Ancient Greek historian Thucydides, may allow an insight for the understanding of current international and national political affairs and international political and economic relations. However, the content of original ancient historical and "journalistic" texts is typically accessible to scholars and other categories of experts, requiring specialized knowledge and command of the ancient language concerned. The present approach targets to facilitate the accessibility of such texts for non-experts in the International Public, especially journalists, translators and students, taking into account basic problems clustered around User Requirements [10]. The possibility of directly accessing the content of these texts and to view features as close as possible to the original text is attempted to be achieved here. Queries based on conventional information extraction strategies may be successful in retrieving concrete information within the "Who/What-When-Where(How)" framework [4] [6], such as names, events (for instance, battles or treaties) and places. However, diplomacy, especially international politics and bilateral relations of the past, mentalities and attitudes of politicians and military men of the past, as well as reactions of citizens to policies in the past constitute complex information that is difficult to be automatically retrieved (Problem 1). An additional problem is that some 
information may be partially or wholly omitted in one target language but may be successfully retained and transferred in another target language, mostly due to the linguistic parameters of the language concerned, but also due to the individual style of the translator (Problem 2). This is of special importance in cases where languages from diverse language families are concerned, such as English, Chinese and Ancient Greek. An additional problem of the texts of Thucydides, as well as other Ancient Greek texts, is the extensive use of pronouns and other forms of anaphora and context-dependent expressions, which pose difficulties for the direct access to information with conventional information extraction methods (Problem 3).

Specifically, the basic issue to be addressed here is the possibility to access complex information in the Ancient Text related to diplomacy and to compare it to passages from online journalistic texts (1) and to directly find out respective passages in the original texts along with a translation in English (2) as well as a second type of translation containing structures close to the original text, minimizing languagespecific interference and parameters of translations (3). The latter possibility (3) provides a closer look to the content and structure of the original text and is less dependent on language-specific parameters interfering in the English translation.

The present approach concerns the integration of expert knowledge within a System-controlled framework for the detection of information concerning diplomacy, especially cause and result relations contained in the online Ancient Text. The module presented here is designed to make use of already-existing tools and mechanisms, the construction of a database and interface with low computational cost, combined with expert knowledge and sublanguage - specific parameters. For the handling of topics related to complex information such as "Diplomacy", expert knowledge and sublanguage - specific parameters are put to use to constitute a framework replacing conventional information extraction methods and statistically-based approaches [1].

\section{Design and User Interaction}

\subsection{Overview and Design}

The proposed approach is designed to work within a framework of a partially implemented interface and database, intending to respond to queries regarding diplomatic and political problems, their resolution, correct or bad decisions, mistakes and socio-cultural phenomena related to politics. The proposed interface and database is specially constructed in respect to the sublanguage of the texts of Thucydides "Peloponnesian War" and is linked to the available translations in English and in formal Modern Greek or "Katharevousa", a "compromise" between Ancient Greek and the Modern Greek used in literature and official documents, especially before the 1980 's. In the present approach, the "Katharevousa" translation plays the role of the so-called "Buffer" translation. Specifically, these translations are the English translation by Welsh writer Richard Crawley (1840-1893) [11] [13] and the "Buffer" translation, namely the translation in Katharevousa Greek by Eleftherios Venizelos [9] [12] converted in English by Google Translate. 
The approach proposed here is based on a set of ontologies interacting with the two corpora, namely the English and Katharevousa Greek translation. These ontologies are sublanguage-based and aided with the additional use of "Universal Words" [7] [8] [14], used within the UNL framework of the United Nations Research project (The UNDL Foundation). The use of "Universal Words" reinforces the access to the multiple keyword search for the International Public, due to the fact that the "Universal Words" are based on a strictly language-independent structure, enabling the processing of languages as diverse as, for example, Chinese, Arabic, Hindi, Japanese, Russian, German, English, French, Portuguese and Greek. The use of "Universal Words" is proposed as an option for the International Users to enable the use of the sublanguage-based ontology and interface in their own native language.

The proposed and partially implemented module may be characterized by a minimal requirement of tools. Specifically, it makes use of the following online tools: (1) the text corpora of the Portal of the Ancient Greek language, of the Institute of the Greek language in Thessaloniki [12], (2) the available online translations of Richard Crawley (English) in websites such as the Internet Classics Archive of the Massachusetts Institute of Technology [13], (3) the online Machine Translation System: Google Translate, as well as (4) "Universal Words" [14].

The present approach concerns a combination of two search mechanisms: the "Buffer" translation and the multiple keyword ontology [1]. The first search mechanism is based on the alignment of the original Ancient Text and the English and Katharevousa Greek ("Buffer") translations, where numbered text passages (average length: 5 - 10 lines) act as pointers to text content.

The second search mechanism is based on the use of multiple keywords derived from both types of corpora, the English translation and the Katharevousa Greek ("Buffer"). The keyword types are English, chosen according to the features of the sublanguage of politics and diplomacy. Specifically, the keywords include proper nouns (including names of persons and places), sublanguage-related terms related to "Facts" (for example "battle", "treaty") as well as sublanguage-related expressions with specific features related to the notion of "Diplomacy". The searched elements also include a group of specified conjunctions constituting pointers to causal relations, such as "because", "due" and sentence-initial "for", as well as conjunctions describing their connection to them, such as "and" and "or".

The search mechanisms presented here are specially engineered for complex types of queries in the sublanguage of politics and diplomacy, since search in respect to simple queries such as basic facts may be restricted only a (English) translation. For complex queries in respect to information related to "Diplomacy", the search is performed on both translations (corpora). In particular, the searched elements are detected at text passage level. When the passage containing the searched elements is detected, the User is presented with the (numbered) passage of the English translation and the Katharevousa "Buffer" translation, with the numbered corresponding passage of the Ancient Text. In particular, the search for the keywords and the respective pointers to Cause-Result relations is designed to be based on maximally four elements 
(sublanguage-specific keywords and UWs) (Figure 1), combined with a Cause-Result conjunction. The multiple words may be retrieved by available software or programmed with the use of the "grep" function [2]. Since the formulation of these concepts may not be easy to access, the dialog box of the interface assists the User to choose the keywords from a list, corresponding to a multiple keyword ontology presented here. The keyword database concerns the multiple keywords derived from the two corpora, the translated English text [11] and the processed Katharevousa Greek "Buffer" translation translation [9]. The sets of keywords are derived from the study of the sublanguage of Thucydides text. The maximal size of the database constructed on ontological principles is designed to comprise about 300 entries.

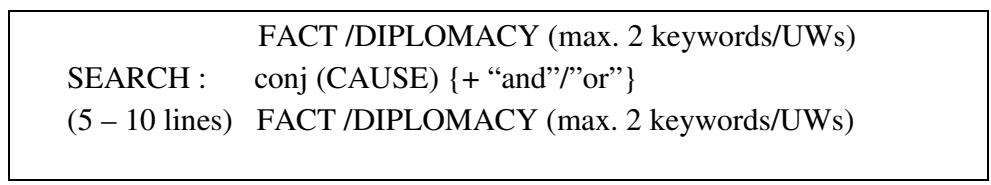

Fig. 1. Search mechanism with multiple keywords

\subsection{User Interaction}

User interaction may be described in two basic steps and an optional step. Specifically, the User is presented with the following features linked to the (a) one or more online journalistic texts obtained from the websites of the news networks: (b) the original Ancient Text, (c) the translations of the original Ancient Text and (d) an interface with queries presenting possible choices to the User. In the first step, the User reads the online journalistic text or texts (a), for instance, in English. The User selects the group of words related to the online journalistic text and types them in the interface of the module (d). Search is performed in respect to passages in the texts containing the search words in the two translations. The words from the User's query are matched to the respective word-group of a keyword database, a multiple keyword ontology constructed with keywords from the English translation and the "Buffer" translation. If there is no match, the module becomes interactive and presents all alternative options (System: "Please select from the following list of words, which best describes your query"). For the International Users who wish to use the sublanguage-based ontology and interface in their own native language, the Universal Words presented in the interface may be used as a stepping stone to access the multiple keywords related to "Diplomacy" and connected to Cause-Result relations and respective passages in the translated and original Ancient Texts.

In the second step, the module presents the passages of the Ancient Text (b) appearing next to the online journalistic text(s). The Ancient Text is presented with the respective English translations (c), namely the available online English [13] translation and the English conversion of the "Buffer" translation of Katharevousa 
Greek, which may contain additional elements not present in the available online English translation, allowing an approximate evaluation of the content from both translations or possible comparison to translations in a third language, other than English. In the optional third step, the User may view the original and the partially edited Greek translation in Katharevousa Greek.

\section{Search Mechanisms}

\subsection{Corpora Alignment}

The first search mechanism concerns an approach including the so-called "Buffer" translation in Katharevousa Greek by the prominent Greek statesman and political leader Eleftherios Venizelos (1864-1936), published in 1940 in the University of Oxford, after his death [9]. The translation is very close to the original Ancient Greek text, however, it explicitly presents most of the information implied by pronouns and other forms of anaphora and context-dependent expressions in the original Ancient Greek text. Thus, the translation by Eleftherios Venizelos [9], provided online by the Portal of the Ancient Greek language, of the Institute of the Greek language in Thessaloniki, Greece [12], is the corpus on which the multiple keyword-based database and link to the English translation is based. It should be stressed that due to the fact that the available translation in Katharevousa Greek minimizes (but does not eliminate) the extensive use of pronouns, other forms of anaphora and contextdependent expressions, it facilitates the direct access to the text content with the use of the sublanguage-specific keywords related to "Diplomacy". We note that in the "Buffer" translation, more causal relations are visible with pointers such as "due" (Figure 2), which might not be available in an original English translation.

For the quick and efficient access to the translation, the translation in English [11] was numbered and matched to the pre-existing number of each corresponding passage in the "Buffer" [9] translation and the linked Ancient Text. The same number is given to the corresponding English translation. This task was a manually performed process requiring a command of English and knowledge of the Katharevousa Greek language. It should be stressed that in the Portal of the Ancient Greek language, the available translation by Eleftherios Venizelos presents marked passages with the same number as the corresponding passages in the original Ancient Text.The aligned English Text and the "Buffer" translation concern the first search mechanism, where search is performed at a numbered text passage level. For the possibility to be processed by Google Translate, the "Buffer" translation was submitted to minimal necessary processing, namely, a partial editing with a simple "replace" function [1] as well as a pre-translation/default correction of selected words related to the "Diplomacy" word group presented below. The editor also starts a new segment with the negation "den" (“ $\delta \varepsilon v ")$, due to observed translation errors by Google Translate. 


\begin{tabular}{|c|}
\hline English Translation: (Crawley, 1903) \\
\hline $\begin{array}{l}\text { [6.24.1] With this Nicias concluded, thinking that he should either } \\
\text { disgust the Athenians by the magnitude of the undertaking, or, if obliged to } \\
\text { sail on the expedition, would thus do so in the safest way possible. }\end{array}$ \\
\hline Google-Translate (Katharevousa text) \\
\hline $\begin{array}{l}\text { [6.24.1] That said Nicias, thinking that due to the number of necessary } \\
\text { supplies will either prevent the Athenians from the campaign or, if forced } \\
\text { to go to war would sail with the utmost safety. }\end{array}$ \\
\hline Minimally preprocessed Katharevousa text \\
\hline 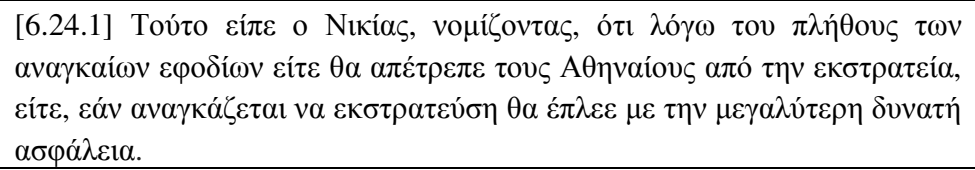 \\
\hline Katharevousa Translation: \\
\hline  \\
\hline Original Ancient Greek Text: \\
\hline 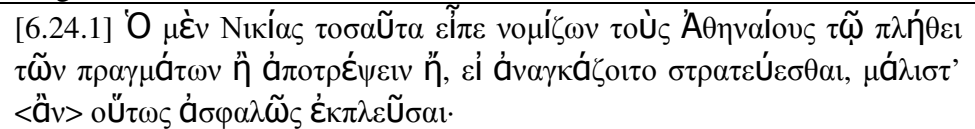 \\
\hline
\end{tabular}

Fig. 2. Aligned Ancient Greek Text and Translations

\subsection{Multiple Keyword Ontology}

The multiple keyword ontology (max. size 300 words) may be divided into two basic categories: (A) Facts and (B) Diplomacy. The category of Facts contains a small group of sublanguage-related keywords related to events such as the concept "war", "battle", "event","incident", "treaty", "ally" "side" and "speech" (approximately 40 words) as well as an open list of proper names, easily accessed with the conventional search using names of people, places and dates [1]. The "Facts" and "Diplomacy" word group are both connected to the Universal Word Framework, whose use is proposed connect sublanguage-specific concepts from both ontologies to each other. The search performed includes the relation of two or more of these concepts in respect to the Facts ("Subject") word group. Two additional word groups are signalized, not belonging to the keyword ontology, namely (a) a set of function words expressing "Cause-Result" relations (conjunctions and adverbials) connecting the "Facts" and/or "Diplomacy" words to each other and (b) a set of words expressing quantity and quality, such as "many", "few", "good", "bad", "large" and "small".

To access and to cover the most commonly occurring types of information related to the subject field of "Diplomacy and other forms of explicitation [3] [5] in the Ancient Text, the category of "Diplomacy" is designed to contain keywords clustered around the concept (Category) (1) state, (2) action and (3) result.The concept of "state" (Category "state") contains singular words or expressions such as "neutrality" 
or "disadvantage". The concept of "actions" (Category "actions") contains expressions such as "response"-"reaction"-"answer" or "accept", "accept", "reject", and "follow". The concept of "result" (Category "result") contains expressions such as "gain""benefit"-"profit" or "loss". The set of verbs contained in this word group are the verbs related to the concepts of feelings and perception "believed", "hoped", "saw" and "feared" (Figure 3). The multiple keyword ontology is enriched with a set of Universal Words, connected to the multiple keywords derived from the two corpora, the translated English text [11] [13] and the processed Greek translation [9]. The Universal Words provide an additional and more language-independent access point to the texts for the International Public. Universal Words may coincide with the multiple "Facts" and "Diplomacy" keywords from the sublanguage (Figure 3).

\begin{tabular}{|c|c|}
\hline UW Example 1 & UW Example 4 \\
\hline 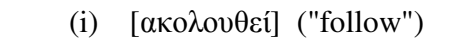 & (i) $[\alpha \pi \mathrm{o} \tau \varepsilon \dot{\lambda} \varepsilon \sigma \mu \alpha]$ \\
\hline UW Example 2 & 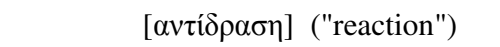 \\
\hline 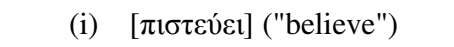 & (ii) $[\alpha \pi \alpha v \tau o v ́ v]$ \\
\hline (ii) $[\beta \lambda \varepsilon ́ \pi \varepsilon 1]$ ("see") & 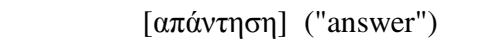 \\
\hline (iii) $[\varepsilon \lambda \pi i \zeta \varepsilon \varepsilon]$ ("hope") & UW Example 5 \\
\hline UW Example 3 & 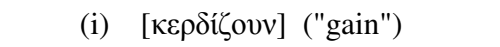 \\
\hline 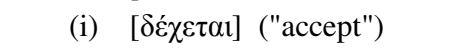 & (ii) $[\varepsilon \pi \omega \varphi \varepsilon \lambda \circ v ́ v \tau \alpha u]$ ("benefit) \\
\hline 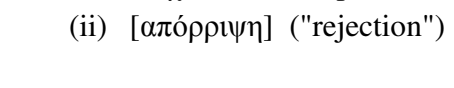 & 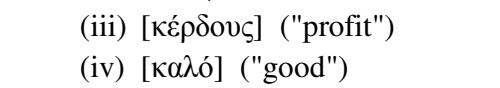 \\
\hline
\end{tabular}

Fig. 3. Greek entries for Universal Words

\begin{tabular}{|c|c|}
\hline $\begin{array}{l}\text { Relation to } U W s 1.1 \\
\text { i. } \quad[\sigma v v \theta \eta \dot{\kappa}\rceil] \text { (“treaty”): } \\
\text { agreement }\end{array}$ & 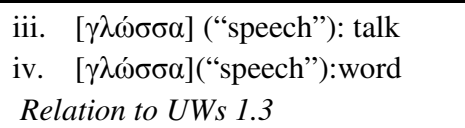 \\
\hline 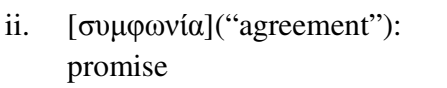 & 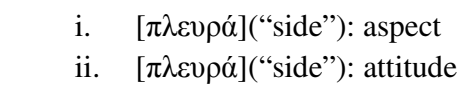 \\
\hline Relation to UWs 1.2 & iii. [ $\pi \lambda \varepsilon v \rho \alpha ́]$ (“side"):opinion \\
\hline $\begin{array}{ll}\text { i. } & {[o \mu \imath \lambda i ́ \alpha](" s p e e c h "): a c t i v i t y} \\
\text { ii. } & {[o \mu \imath \lambda i ́ \alpha] \text { (speech): information }}\end{array}$ & 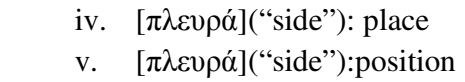 \\
\hline
\end{tabular}

Fig. 4. Relation of Universal Words with Facts and Diplomacy Word Groups

The Facts and Diplomacy word groups are compatible with the Universal Word Framework, whose use is proposed to connect sublanguage-specific concepts from both ontologies to each other. Therefore, there is a connection between a general (or universal) ontology, in this case, the Universal Word framework, and a sublanguagespecific ontology. Universal Words are designed to be compatible with a number of languages, aiming to provide a language-independent analytical framework. This framework is especially helpful in languages with a remarkable polysemy of commonly occurring concepts, such as Ancient Greek, since it allows a concept in the 
form of a Universal Word to be directly connected to the ontology designed for the sublanguage. The Universal Word framework concerns concepts already connected to each other, such as "treaty" classified as "agreement", also related to the concept "promise", the concept of "speech" connected to "activity" and "information", as well as the concept of "side" connected to "opinion", "attitude" and "aspect", "place" and "position" (Figure 4). Examples of such connected concepts within the sublanguage-specific "Diplomacy" word group are the relations of such as "believe" connected to "hope", "reaction" connected to "response", "benefit" connected to "useful" and "loss" connected to "disadvantage" (Figure 3). Other examples within the Universal Word framework are "fear" classified as "feeling", "answer" classified as "information" and "follow" containing the concept "watch" in its encoding.

\section{Examples of Accessing Cause-Result Relations}

In the following examples of accessing Cause-Result relations we note that the online journalistic texts accessed from the international news networks are not presented here, to avoid any connection to sensitive political issues in international affairs. The User may wish to acquire information in respect to various queries, for example, the concepts "benefit from neutrality" or "hope to become leader" or "change sides". In the "multiple ontology" from both corpora (translations) and the UWs, there is a direct match to the User's query, for example, in passage 5.28.2 (Figure 5) in respect to the words "neutrality" to the Category "state" and "benefit" related to the Category "result".

\begin{tabular}{|c|c|}
\hline \multirow[t]{2}{*}{ Online Texts: } & ONLINE JOURNALISTIC TEXT-1: text-text- text-text- text-text- text-text- text-text- text- \\
\hline & ONLINE JOURNALISTIC TEXT-2: text-text- text-text- text-text- text-text- text-text- text- \\
\hline English Translation: & http://classics.mit.edu/Thucydides/pelop \\
\hline \multicolumn{2}{|c|}{$\begin{array}{l}\text { [5.28.2] Argos came into the plan the more readily because she saw that war with Lacedaemon was inevitable, the } \\
\text { truce being on the point of expiring; and also because she hoped to gain the supremacy of Peloponnese. For at this time } \\
\text { Lacedaemon had sunk very low in public estimation because of her disasters, while the Argives were in a most } \\
\text { flourishing condition, having taken no part in the Attic war, but having on the contrary profited largely by their } \\
\text { neutrality. }\end{array}$} \\
\hline \multicolumn{2}{|l|}{ Google-Translate: } \\
\hline \multicolumn{2}{|c|}{$\begin{array}{l}\text { The Argos showed so much more uplifting to follow this policy because they saw that the imminent expiry of the } \\
\text { Spartans after the Treaty of the round such war was inevitable, and while it captured the hope that it will become head } \\
\text { of the Peloponnese. Because at that time too brought against Lacedaemon, and prestige have been forfeited due to } \\
\text { mishaps, while the Argos are in excellent position in any respect, because not shared the burden of the war with } \\
\text { Athens, and took place in peace to both parties benefited from interest contrary hence. }\end{array}$} \\
\hline & http://www.greeklanguage.gr/greekLang/ancient_greek/tools/corpora/anthology/ \\
\hline \multicolumn{2}{|c|}{ 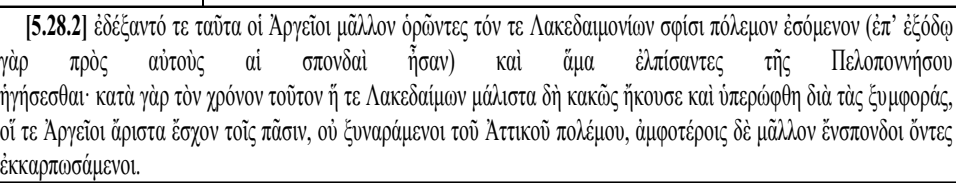 } \\
\hline
\end{tabular}

Fig. 5. Interface with retrieved passage and translations from query 
A related word to the Category "result" is the word "interest". The concept "hope to become leader" is matched to the verb "hope" and the sublanguage-related keyword "leader". A related word is the word "supremacy" (Category "state"). The words "condition", "position" and "prestige" in the passage accessed are also included in the Category "state". To indicate Cause-Result relations, the "for" and "because" causal conjunctions are signalized, appearing in passages of both translations. However, certain User queries cannot be directly matched to the keywords. In the present case, there is no direct match to the concept "change sides", since this type of expression was not typical of Thucydides. In this case, the search process becomes more interactive. The System proposes the concept matched to the keyword "side" in the Category "state" of the keyword ontology. The System also presents the list with the closest matching multiple keywords. The User chooses the word "follow" from the Category "action" and the sublanguage-related keyword "side". However, no match is found. A match in the same passage 5.28.2 is achieved with keywords "follow" and "policy" (Figure 5).

In Figure 6, the User may wish to acquire information in respect to the query "outcome of war is unpredictable", "small unrests may lead to major outbreaks" or "failure due to underestimating smaller opponent" or "lack of preparation". The concepts are matched, for example, in the passage 2.11.4 (Figure 6) in respect to the keyword "war" and the concepts "outcome" ("fortune", "unpredictable" in Category

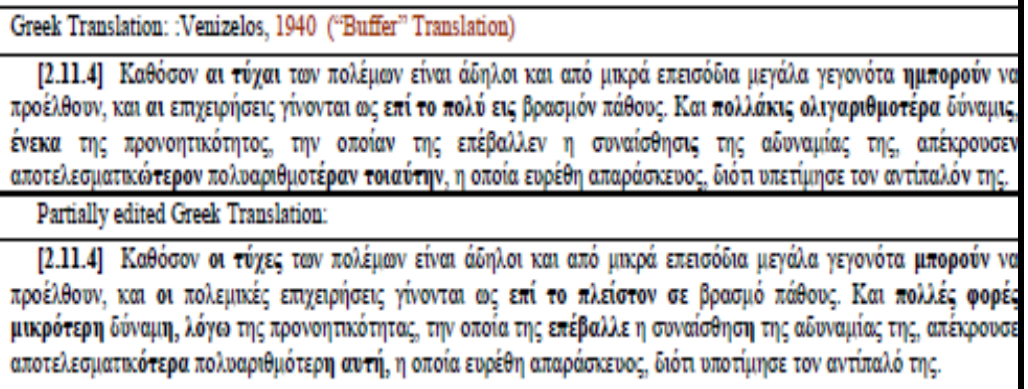

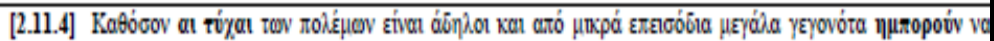

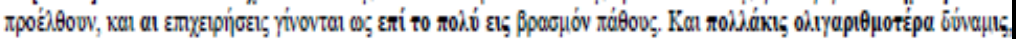

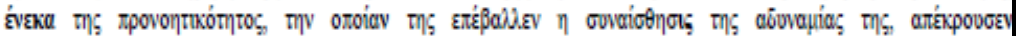

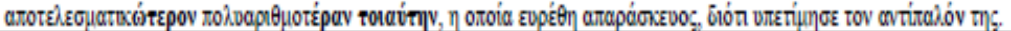

Partially edited Greek Translation:

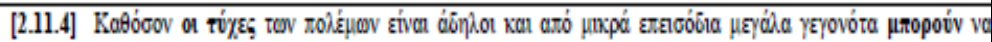
тров́

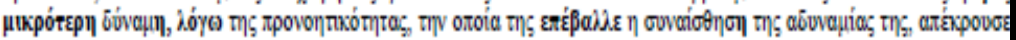

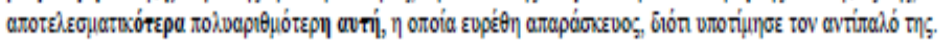

"The course of war cannot be foreseen, and its attacks are generally dictated by the impulse of the moment; and where overweening self-confidence has despised preparation, a wise apprehension often been able to make head against superior numbers". [English (Crawley) Translation]

"As the fortunes of war are hidden and small events big events can be generated, and military operations are mostly in a boiling passion. And many times less power, because of anticipation, of which impose a sense of failure, effectively repelled more numerous, which was found unprepared because they underestimated her opponent" ["Buffer" Translation- Google Translate]

Fig. 6. Retrieved passage and translations from query 
"result"), and the verbs "predict" and "forsee" retrieved from both corpora. The second query is matched to the sublanguage-related term "unrest" related to the keyword "event", as well as the words "small" and "major" related to quantity and quality. For the third query, there is an additional match in passage 2.11 .4 to the words "confidence" and "unprepared" (Category "state"), the word "failure" (Category "Result"), as well the verb "underestimate". The Cause-Result relations are signalized by the causal "as" and the "because" conjunctions, appearing in passages of the "Buffer" translation, connected to each other with the "and" conjunction.

\section{Conclusions and Further Research}

Although the proposed module may not provide an in-depth analysis of the Thucydides text, it intends to capture the most commonly occurring categories of diplomatic information and to provide access related to most types of information related to Cause-Result relations. Expert knowledge of the translations provided by R. Crawley (a writer), and E. Venizelos (a politician) is provided, as well as knowledge of Katharevousa Greek texts, containing elements close to Ancient Greek. Thus, complex information such as diplomacy is handled both by expert knowledge and sublanguage - specific parameters, replacing statistically-based approaches and allowing the use of already-existing tools, a database and interface with low computational cost. Further research and full implementation by User groups may provide more upgraded versions of the present design and evaluation results for further development. We note that the tool could have an even simpler configuration if online Machine Translation of Katharevousa Greek were available. Furthermore, these specifications may be adapted to the needs of Ancient Texts in other languages.

\section{References}

1. Alexandris, C.: User Interface Design for the Interactive Use of Online Journalistic Texts and Ancient 'Journalistic' Texts for the International Public. In: Proceedings of the International Conference on Applied and Theoretical Systems Research (ATISR) 2012, Taipei, Taiwan (2012)

2. Bambenek, J., Klus, A.: Grep Pocket Reference. O'Reilly, Sebastopol (2009)

3. De Silva, R.: Explicitation in Media Translation: English Translation of Japanese Journalistic Texts. In: Proceedings of the 1st Portuguese Translation Conference, Caparica, Portugal (2006)

4. Gaizauskas, R., Foster, J., Wilks, Y., Arundel, J., Clough, P., Piao, S.: The METER Corpus: A Corpus for Analysing Journalistic Text Reuse. In: Proceedings of the Corpus Linguistics 2001 Conference, March 29-April 2, Lancaster University, United Kingdom (2001)

5. Hatim, B.: Communication Across Cultures: Translation Theory and Contrastive Text Linguistics. University of Exeter, Exeter (1997)

6. Jurafsky, D., Martin, J.: Speech and Language Processing, an Introduction to Natural Language Processing, Computational Linguistics and Speech Recognition, 2nd edn. Prentice Hall series in Artificial Intelligence. Pearson Education, Upper Saddle River (2008) 
7. Uchida, H., Zhu, M., Della Senta, T.: Universal Networking Language. The UNDL Foundation, Tokyo (2005)

8. Uchida, H., Zhu, M., Della Senta, T.: The UNL, A Gift for Millennium. The United Nations University, Institute of Advanced Studies UNU/IAS, Tokyo, Japan (1999)

9. Venizelos, E.: Thoukudidou Istoriai: Kata Metaphrasin Eleutheriou Benizelou. Thucydides' History: translated by Eleftherios Venizelos, ed. D. Caclamanos, 2 vols. Oxford University Press, Oxford (1940)

10. Wiegers, K.E.: Software Requirements. Microsoft Press, Redmond (2005)

11. Thucydides' Peloponnesian War, translated by Richard Crawley. J.M. Dent and Co., London (1903)

12. Centre for the Greek language: Portal for the Greek Language: http://www.greeklanguage.gr, E.Venizelos Translation [1940] 1960.

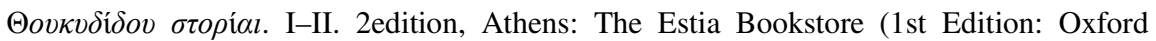
University Press) (in Greek)

13. Internet Classics Archive of the Massachusetts Institute of Technology: http: / / classics.mit.edu/Thucydides/pelopwar.mb.txt

14. The Universal Networking Language: http: / / www . undl . org 